AIAA-2001-1320

\title{
FUZZY STRUCTURES ANALYSIS OF AIRCRAFT PANELS IN NASTRAN
}

\author{
Victor W. Sparrow* \\ The Pennsylvania State University, University Park, PA 16802 \\ Ralph D. Buehrle ${ }^{+}$ \\ NASA Langley Research Center, Hampton, VA 23681
}

\begin{abstract}
This paper concerns an application of the fuzzy structures analysis (FSA) procedures of Soize to prototypical acrospace panels in MSC/NASTRAN, a large commercial finite element program. A brief introduction to the FSA procedures is first provided. The implementation of the FSA methods is then disclosed, and the method is validated by comparison to published results for the forced vibrations of a fuzzy beam. The results of the new implementation show excellent agreement to the benchmark results. The ongoing effort at NASA Langley and Penn State to apply these fuzzy structures analysis procedures to real aircraft panels is then described.
\end{abstract}

\section{Introduction}

In 1986 Christian Soize was at the Office National d'Études et de Recherches Aérospatiales (ONERA) ${ }^{1}$ in Chatillon, France and introduced the concept of fuzzy structures ${ }^{2,3,4,5,6}$ in the dynamic analysis of large scale structures. Having nothing to do with the use of fuzzy sets or fuzzy logic,

* Associate Prof., Grad. Program in Acoustics. Email: sparrow@helmholtz.acs.psu.edu. Member AIAA.

+ Aerospace Engincer, Structural Acoustics Branch. E-mail: r.d.buehrle@larc.nasa.gov.

Copyright (C) 2001 by the American Institute of Aeronautics and Astronautics, Inc. No copyright is asserted in the United States under Title 17, U.S. Code. The U.S. Government has a royalty-free license to exercise all rights under the copyright claimed herein for Governmental purposes. All other rights are reserved by the copyright owner. a fuzzy structure is composed of (1) a well characterized master structure and (2) attached fuzzy substructures whose details are either unknown or are imprecisely known. In the context of a traditional finite element model, the master structure would be that part of the structure amenable to conventional FEs. The fuzzy substructure would be those parts of the structure inaccessible to traditional modeling: missing degrees of freedom, fine scale structural details, attached equipment, etc. An implementation of the fuzzy structures concepts in FEs as originally conceived by Soize is called Fuzzy Structures Analysis (FSA), a term coined by Ruckman. $^{7}$

Although there have been some attempts to implement alternative fuzzy structure approaches in the context of analytical modeling, $8,9,10,11,12$ the original FSA methodology of Soize has great attractiveness since it can be performed using existing $\mathrm{FE}$ models: no new special elements need be generated. What las been lacking, however, is an implementation of FSA in a mainstream FE code.

This paper describes an original implementation of Soize's FSA in the well known commercial code MSC/NASTRAN ${ }^{13}$ specifically its solution sequence 108, the dynamic frequency response module. Using NASTRAN's Direct Matrix Abstraction Program (DMAP) environment and a MATLAB ${ }^{14}$ module to provide appropriate input parameters, the new implomentation allows one to include fuzzy substructures along with any structural model already existing in NASTRAN. The new DMAPs have been benchmarked against published results for vibrations of beams with attached fuzzy substructures, and these benchmarks are reported here for the first time. This paper also describes an ongoing application of the new FSA implementation to two representative aircraft fuselage panels under

American Institute of Aeronautics and Astronautics 
study at the NASA Langley Research Center.

This paper is arranged with the following sections: Firstly, the background of Soize's fuzzy structures analysis is provided, focusing on both the overall concepts of the method and the required mathematical formulation. Secondly, this paper describes the specific implementation of the fuzzy structures analysis procedures in MSC/NASTRAN and MATLAB. Next a benchmark solution is described that was used to test the procedures. Two aircraft panels are then described which are the focus of our ongoing study in applying the fuzzy structures analysis procedures to real aircraft panels.

Note that the acronym FSA is used for fuzzy structures analysis throughout this paper. Also for consistency with Soize's works, the $e^{+i \omega t}$ time convention is used.

\section{Background of FSA}

\section{Motivation}

Soize introduced fuzzy structures for modeling systems with a huge number of degrees of freedom with a reduced size model. A good example of a fuzzy structure, a system too complicated for conventional modeling, would be the electronics under the cockpit of a commercial airliner. This mechanical system cannot be modeled directly because it is so complex. However, it must be modeled somehow since it contributes to the overall dynamics and structural acoustic response of the airplane. This particular fuzzy structure is important for interior cabin noise upon landing since it may be directly above or near the forward landing gear of the airliner, and the landing gear vibrations can travel along the fuselage and cause acoustic radiation into the cabin.

Another aim for FSA procedures is to account for uncertainty of small scales in structural dynamics models. One source of uncertainty is in the variability in the construction of large airliners. Another source of uncertainty is in the finite element modeling procedure itself, since no individual can possibly explicitly incorporate into a deterministic finite element model all of the fine structure in a realistic airliner model. One idea put forth by Jeffrey Cipolla ${ }^{15}$ in 1995 was to think of FSA as a large eddy simulation (LES) for large dynamics problems in analogy with how fluid dynamicists handle fluid scales too small for explicit representation in a direct numerical simulation (DNS).

The computational cost is low for FSA compared with the conventional Monte Carlo modeling of uncertainties in finite element simulations. In 1986 Soize showed one could achieve similar results between a FSA and a traditional Monte Carlo model of an uncertain structure but with many fewer FE solves. ${ }^{2}$ This computational savings, along with keeping only a minimum number of DOFs in the FE model, is the great advantage of FSA.

\section{Previous work}

The algorithm for implementing Soize's FSA for complicated structures has been available in the literature for many years. A somewhat complicated algorithm to be described briefly below, the procedure involves making a few runs of a conventional structural dynamics finite element analysis program with a large number of carefully constructed right hand sides (forcing functions). The results obtained using each of the forcing functions is then handled using standard statistical techniques.

In 1994 the Carderock Division of the Naval Surface Warfare Center tasked one engineer, Chris Ruckman, to travel to France to investigate Soize's fuzzy structures methods in detail. One of Ruckman's goals was to determine the feasibility of implementing the fuzzy structures procedures in NASTRAN, and he wrote a short report ${ }^{16}$ on how he thought one could accomplish this. He also published an even shortened version of the report as a paper at conference. ${ }^{7}$ After the work was written up, changing priorities and reduced budgets at NSWC and the Office of Naval Research stopped further development of implementing the fuzzy structures procedures in NASTRAN. Ruckman left NSWC, and he moved on to other areas. However, Ruckman correctly predicted that a fuzzy structures analysis in NASTRAN was possible.

At about the same time the company now known as BBN Technologies of Cambridge, MA also was tasked by the Office of Naval Research to include a fuzzy finite element capability in their program SARA, a coupled fluid and structure finite element solver. ${ }^{17}$ This development was accomplished in 1994 using the Pierce/Sparrow/Russell ${ }^{8}$ and Strasberg/Feit ${ }^{12}$ fuzzy structure models instead of Soize's model. Because of reduced budgets, however, no detailed testing or validation of the BBN implementation, to the best of our knowledge, was 
ever made. SARA is not used in the commercial aircraft industry. However, since NASTRAN is more widely in use, the present authors decided that the prudent course was to implement Soize's procedures in NASTRAN, putting them into the mainstream for testing and validation by NASA Langley, industry, and university researchers.

\section{Philosophy}

The general philosophy of FSA is to model as much of structure/flud with conventional finite elements as possible. This portion of the structure, accessible to conventional modeling techniques is called the master structure. To add the influence of additional fine detail or uncertain attachments to the master structure, one modifies the boundary conditions of the master structure. See Fig. 1, where the $Z$ 's indicate boundary impedances affecting the master structure. This addition is called the fuzzy substructure. By only incorporating the details of the fine or uncertain structure through the boundary conditions only, the total number of degrees of freedom (DOFs) in the model is unchanged. This helps keep the size of the finite element model from increasing to a point where conventional FE solvers are unusable.

It should be noted that the fuzzy substructure, in the present analysis, only affects the master structure through the FE nodes. One only places the fuzzy substructure at nodes where one expects the uncertainty or fine structure to exist.

\section{Fuzzy impedances}

Each additional boundary condition in the fuzzy substructure is modeled as the impedance of a one degree of freedom attachment. See Fig. 2 where the mass $\mu$ is connected to the master structure through a stiffness $K$ and a damping constant $C$ and where $\omega_{p}=(\mathrm{K} / \mu)^{\frac{1}{2}}$ is the natural frequency. Here $\xi=\frac{\mathrm{C}}{2 \mu \omega_{p}}$ is the critical damping constant assumed to be small, $(0<\xi<1)$. Assuming the support has a normal velocity $\dot{x}=v=\hat{v} e^{i \omega t}$ with driving frequency $\omega$, one can easily show

$$
Z=\frac{\hat{F}}{\hat{v}}=\frac{-\mu \omega_{p}^{2}\left(\frac{\omega_{p}^{2}}{\omega^{2}}-1+4 \xi^{2}\right)+i \omega\left(2 \mu \xi \omega_{p}\right)}{i \omega\left[\left(1-\frac{\omega_{p}^{2}}{\omega^{2}}\right)^{2}+4\left(\frac{\omega_{p}^{2}}{\omega^{2}}\right) \xi^{2}\right]}
$$

for the impedance of the attachment.

To make the oscillator fuzzy/uncertain the following assumptions are made about the mass, damping, and modal density $n$ of the oscillator:

$$
\begin{aligned}
\mu & =\underline{\mu}\left(1+Y_{1}\right) \\
\xi & =\underline{\xi}\left(1+Y_{2}\right) \\
n & =\underline{n}\left(1+Y_{3}\right)
\end{aligned}
$$

where _ indicates mean value, and where the $Y_{i}$ are independent random variables, in practice small fractions of unity. ${ }^{18}$

A one DOF oscillator having modal density may be unfamiliar, but it simply is equivalent to the oscillator having uncertainty in natural frequency $\omega_{p}$

\section{Incorporation into the dynamic model}

In the following paragraphs $\bar{U}$ indicates a column vector of generalized displacements, $\bar{F}$ a column vector of generalized forces, and square brackets [] indicate matrices. A conventional structural dynamics vibration problem would be formulated for a particular angular frequency $\omega$ using the FE equation

$$
i \omega\left(\left[Z_{\text {master structure }}\right]\right) \bar{U}=\bar{F}(\omega) .
$$

Adding the effect of the fuzzy substructure consists of modifying the dynamical equation at appropriate nodes as

$$
i \omega\left(\left[Z_{\text {master structure }}\right]+\left[Z_{\mathrm{fuzzy}}\right]\right) \bar{U}=\bar{F}(\omega)
$$

Decomposing the fuzzy substructure into its mean and random components one has $\left[Z_{\text {fuzzy }}\right]=[\underline{Z}]+$ $\left[Z_{\text {rand }}\right]$ yielding

$$
i \omega\left(\left[Z_{\mathrm{det}}\right]+\left[Z_{\mathrm{rand}}\right]\right) \bar{U}=\bar{F}(\omega)
$$

for the dynamical system, where $\left[Z_{\mathrm{dct}}\right]=$ $\left[Z_{\text {master structure }}\right]+[\underline{Z}]$. Soize then pulls out $\left[Z_{\mathrm{det}}\right]$,

$$
i \omega\left[Z_{\mathrm{det}}\right]([I]-[T]) \bar{U}=\bar{F}(\omega),
$$

an equation which can be solved by a perturbation analysis. Here $[T]=-\left[Z_{\mathrm{dct}}\right]^{-1}\left[Z_{\text {rand }}\right]$ has entries assumed small compared to unity. Soize defines

$$
\left[Z_{\text {rand }}\right]=\sum_{i=1}^{M} \chi_{i}\left[Z_{i}\right]
$$

where the $\left[Z_{i}\right]$ are the contributions to $\left[Z_{\text {rand }}\right]$ by each random aspect of the fuzzy. There is 
only 1 non-zero element in each $\left[Z_{i}\right]$. There are $M=L \times J \times d$ contributions to the sum:

$L=$ number of attachments

$J=$ number of directions ( 3 in general)

$d=$ number of fuzzy parameters (usually $3-4$ ) and the $\chi_{i}$ are independent, centered, uniform random variables. Note that the construction of the $\left[Z_{i}\right]$, too lengthy to give here, is completely described in the works of Soize $\mathrm{e}^{2,3,4,6}$ as are other details concerning the solution procedure.

Formally, if it could be constructed, the solution of Eq. (6) is

$$
\bar{U}=([I]-[T])^{-1}\left(i \omega\left[Z_{\mathrm{det}}\right]\right)^{-1} \bar{F}(\omega) .
$$

Because $\|T\|<1$ one can write

$$
([I]-[T])^{-1}=[I]+\sum_{k=1}^{\infty}[T]^{k}
$$

So Eq. (8) becomes

$$
\bar{U}=\left([I]+\sum_{k=1}^{\infty}[T]^{k}\right)\left(i \omega\left[Z_{\mathrm{det}}\right]\right)^{-1} \bar{F}(\omega)
$$

where

$$
[T]=-\left[Z_{\mathrm{dct}}\right]^{-1} \sum_{i=1}^{M} \chi_{i}\left[Z_{i}\right]
$$

The solution is constructed by expanding out Eq. (10):

$\bar{U}=\bar{U}^{(0)}+\sum_{i=1}^{M} \chi_{i} \bar{U}_{i}^{(1)}+\sum_{i_{1}=1}^{M} \sum_{i_{2}=1}^{M} \chi_{i_{1}} \chi_{i_{2}} \bar{U}_{i_{1} i_{2}}^{(2)}+\ldots$

Each of the $\bar{U}^{(k)}$ are solved for easily, and each of these solves is completely deterministic. The $\chi_{i}$ are the only random variables in Eq. (12).

\section{Solution sequence}

The usual solution sequence follows from solving Eq. (10) with increasing orders of the index $k$ in these steps:

0 . FE solve with master structure and mean fuzzy only.

1. Another FE solve of master structure with specially constructed right hand sides (RHSs) using results of 1 st solve (Step 0).

2. If necessary, another FE solve of master structure using specially constructed RHSs using results of 1 st and 2 nd solves (Steps 0 and 1 ).
3. Postprocessing of results gives mean and variance of response.

Here are additional details:

Step Q: $k=0$. The initial step gives $\bar{U}^{(0)}$ by solving

$$
i \omega\left[Z_{\mathrm{det}}\right] \bar{U}^{(0)}=\bar{F}(\omega)
$$

This is equivalent to solving Eq. (10) omitting the $\sum_{k=1}^{\infty}[T]^{k}$ sum completely. Note that the solution of Eq. (13) is completely deterministic.

Step 1: $k=1$. Get $\bar{U}_{i}^{(1)}$ for $1 \leq i \leq M$ by solving

$$
i \omega\left[Z_{\mathrm{det}}\right] \bar{U}_{i}^{(1)}=-i \omega\left[Z_{i}\right] \bar{U}^{(0)} .
$$

This is equivalent to solving Eq. (10) keeping only the first term of the $\sum_{k=1}^{\infty}[T]^{k}$ sum. Usually Eq. (14) is implemented using 1 solve with $M$ RHSs for each frequency. Each frequency must be solved separately since $\left[Z_{\text {det }}\right]$ turns out to be frequency dependent. Further, this $k=1$ solve, again completely deterministic, usually is enough to represent $\mathrm{Eq}$. (12) with sufficient accuracy.

Step 2: $k=2$. If necessary one further solves for $\bar{U}_{i_{1} i_{2}}^{(2)}$ for $1 \leq i_{1} \leq M$ and $1 \leq i_{2} \leq M$ by solving

$$
i \omega\left[Z_{\mathrm{det}}\right] \bar{U}_{i_{1} i_{2}}^{(2)}=-i \omega\left[Z_{i_{2}}\right] \bar{U}_{i_{1}}^{(1)}
$$

This is equivalent to solving $\mathrm{Eq}$. (10) keeping the first two terms of the $\sum_{k=1}^{\infty}[T]^{k}$ sum. Here Eq. (15) is implemented using 1 deterministic solve with $M^{2}$ RHSs. Since a solve with $M^{2}$ RHSs could be prohibitively expensive, one hopes that this step is not necessary.

\section{Step 3, Postprocessing:}

Now that the $\bar{U}_{i}^{(1)}$, and if necessary $\bar{U}_{i_{1} i_{2}}^{(2)}$, components of $\mathrm{Eq}$. (12) have been found, one can find the statistics of the solution. Ruckman ${ }^{7,16}$ elucidated this procedure.

Clearly the solution is in the form of generalized displacements and the expectation (average) of $\bar{U}$ is simply $\bar{U}^{(0)}$ obtained in step 0 of the solution sequence. However, the expectation of any magnitude squared value is

$$
E\left\{|r|^{2}\right\}=\left|r^{(0)}\right|^{2}+\sum_{i=1}^{M}\left|r_{i}^{(1)}\right|^{2}
$$


for $k=1$ when $r$ is any scalar value such as displacement, velocity, acceleration, pressure, etc. The variance is further given for $k=1$ as

$$
\begin{aligned}
V\left\{|r|^{2}\right\}= & 4 \sum_{i=1}^{M}\left[\operatorname{Re}\left\{r^{(0)} r_{i}^{*(1)}\right\}\right]^{2}-\frac{6}{5} \sum_{i=1}^{M}\left|r_{i}^{(1)}\right|^{4} \\
& +2 \sum_{i=1}^{M} \sum_{j=1}^{M}\left[\operatorname{Re}\left\{r_{i}^{(1)} r_{j}^{*(1)}\right\}\right]^{2}
\end{aligned}
$$

\section{Implementation}

\section{Overview}

To implement the solution procedure just described it was decided to use two consecutive NASTRAN DMAP runs, including modifications to the SOLUTION 108 (direct frequency response) module. The NASTRAN database capabilities would be used to minimize matrix recalculations between the two runs. The first NASTRAN DMAP run saves all the necessary values through a simple execution of the usual 108 sequence, performs precalculations, and saves needed information to the NASTRAN database. The second NASTRAN DMAP run actually executes the $k=0$ and $k=1$ steps in the solution sequence described above as well as performing the solution postprocessing. An extensive MATLAB script is executed after the first NASTRAN run to precalculate information for the $\left[Z_{i}\right]$ matrices, required for the second NASTRAN run.

The first DMAP code consists of 123 lines of DMAP inserted into usual SOLUTION 108 (direct frequency response) bulk data file. Here is where the usual 108 sequence is used. It also saves the database and counts the number of fuzzy DOFs. The second DMAP code contains over 650 lines of DMAP. It uses the NASTRAN database saved in the first DMAP, but is essentially a stand alone analysis.

The MATLAB code, "makefuzzy," provides inputs for the second DMAP code since NASTRAN can't perform 2-D numerical integration, required for generating the $\left[Z_{i}\right]$ matrices.

Details on FSA DMAP 2

Usually NASTRAN represents the mass, damping, and stiffness matrices straightforwardly as

$$
i \omega\left[Z_{\mathrm{det}}\right]=-\omega^{2}[M]+i \omega[D]+[K]
$$

However, in FSA the damping and mass matrices become frequency dependent as

$$
\begin{aligned}
i \omega\left[Z_{\mathrm{dct}}\right]= & -\omega^{2}\left([M]+\left[R_{\mathrm{fuz}}(\omega)\right]\right) \\
& +i \omega\left([D(\omega)]+\left[I_{\mathrm{fuz}}(\omega)\right]\right)+[K(\omega)]
\end{aligned}
$$

as they now include the real and imaginary contributions of the fuzzy substructure, $\left[R_{\mathrm{fuz}}(\omega)\right]$ and $\left[I_{\mathrm{fuz}}(\omega)\right]$, respectively.

Hence the FSA DMAP 2 must solve for one frequency at a time. Note that this is not NASTRAN's default procedure. Usually MSC/NASTRAN attempts to perform the direct frequency response for all frequencies in solution 108 during the same solver pass.

To explicitly clarify what has to occur, a solve for the lst frequency would take the form $i \omega\left[Z_{\mathrm{det}}\left(\omega_{1}\right)\right] \bar{U}^{(0)}=\bar{F}\left(\omega_{1}\right)$, then one solves

$$
i \omega\left[Z_{\mathrm{det}}\left(\omega_{1}\right)\right] \bar{U}_{i}^{(1)}=-i \omega\left[Z_{i}\left(\omega_{1}\right)\right] \bar{U}^{(0)}\left(\omega_{1}\right)
$$

for each $i$. Similarly, the corresponding solve for the 2nd frequency would be $i \omega\left[Z_{\mathrm{det}}\left(\omega_{2}\right)\right] \bar{U}^{(0)}=\bar{F}\left(\omega_{2}\right)$, then one solves

$$
i \omega\left[Z_{\text {det }}\left(\omega_{2}\right)\right] \bar{U}_{i}^{(1)}=-i \omega\left[Z_{i}\left(\omega_{2}\right)\right] \bar{U}^{(0)}\left(\omega_{2}\right)
$$

again for each $i$, etc.

User procedure

When running a typical FSA, the following procedure is followed:

- Run FSA DMAP 1

- (use scr=no command line option)

- Input: nodes designated as fuzzy in a SET

- Output: DBALL database file

- Runtime: about same as SOLUTION 108

- Run Matlab Code "makefuzzy"

- Input: frequencies for analysis, fuzzy parameters

- Output: file fuzzyforphase1a2.txt, matrix element values

- Runtime: 5 min on a DEC Alpha workstation for 100 frequencies.

- Run FSA DMAP 2

- Input: fuzzyforphase la2.txt, "FSA DMAP 1" .DBALL file

- Output: mean squared displacement, etc 
- Runtime: $2 \times$ (SOLUTION 108 runtime) + time for RHS calculations.

The number of RHSs $=9 \times$ number of fuzzy nodes. This is because in the present implementation only displacement DOFs (3) are assumed to be fuzzy, and only 3 fuzzy variables are assumed for each DOF. The 3 fuzzy variables are mass, critical damping, and modal density given in Eq. (2). The amount of deviation for these variables from their mean values are specified by the user as dispersion parameters $\lambda_{1}, \lambda_{2}, \lambda_{3}$ in the MATLAB procedure "makefuzzy."

\section{Testing and validation}

The resulting DMAP/Matlab procedures have been successfully applied to a benchmark problem published by Soize, a fuzzy simply supported beam. ${ }^{5}$

\section{Model parameters}

The base beam (master structure) parameters were taken directly from Soize's work ${ }^{5}$. The beam mass was $20,000 \mathrm{~kg}$, and the attached oscillators have a 0.002 damping rate and a mass of $1.8 \mathrm{~kg}$. The dispersion parameters were matched to be $\lambda_{1}=\lambda_{2}=\lambda_{3}=0.001$.

However, the beam modeled here did have some differences to Soize's beam. Soize's model had $2500 \mathrm{FE}$ nodes and was modeled using a $0.05 \mathrm{~Hz}$ frequency resolution. He used 1213 1-DOF fuzzy attachments in the $y$ ("up") direction, with $\underline{n}=$ $0.035,0.043$ for mean modal density on two distinct patches of oscillators. In the present work only a $200 \mathrm{FE}$ node model was employed using a $1 \mathrm{~Hz}$ frequency resolution from 1 to $100 \mathrm{~Hz}$. Here 300 1-DOF fuzzy attachments were distributed over the two patches specified in Soize's problem, but they were in all 3 directions $(x, y$, and $z)$ and they all used the same value, 0.035 , for mean modal density. The 300 fuzzy attachments were located at 100 fuzzy nodes, corresponding to 900 RHSs in the $k=1 \mathrm{FE}$ solution. In summary for the present FSA DMAP 2, $100 k=1$ individual FE solves were made, 1 solve for each frequency, with each solve containing the 900 RHSs.

The run times on a NASA Langley Structural Acoustics Branch DEC Alpha workstation were

- 1 minute - FSA DMAP 1

- 5 minutes - "makefuzzy" Matlab file

- $6 \mathrm{~h}, 7$ minutes - FSA DMAP 2
Clearly the codes are not yet optimized for spced, but these numbers provide some evidence of the substantial time required for the procedure.

$\underline{\text { Results }}$

The plot shown in Fig. 3 clearly indicates the similarity of the results for the mean squared acceleration at a point on the beam published as Fig. 7 (a) of Soize. ${ }^{5}$ Here the solid line gives the fuzzy structures result and the dashed line gives the result for the master structure alone. (The additional thin solid line in Soize's Fig. 7(a) is one realization of a Monte Carlo simulation for comparison.) Sparrow's beam model has a coarser discretization (200 FE nodes) and frequency resolution compared to Soize's model (2500 FE nodes), but otherwise the plots agree very well in magnitude. A close inspection of the two results shows that there is a slight discrepancy in the location of the resonances, and this again is due to the different discretizations of the models. These results clearly indicate that these new fuzzy structures procedures in DMAP are working correctly and are ready for application to the more realistic panel structures of interest to the aerospace and transportation industries.

Note also in Fig. 3 the greatly constricted fluctuations of the structure's acceleration with the addition of the fuzzy substructure. In essence, the fuzzy substructure acts to damp the master structure vibrations.

\section{Prototypical aircraft pancls}

The present research at Penn State and NASA Langley is now focused on applying the new FSA procedures to realistic, prototypical aircraft panels. The investigation is centered on modeling the junctions between the longerons and skin and frames and skin in such panels as fuzzy/uncertain due to the inherent impreciseness of such joints. Note that this is a departure from Soize's original application of the FSA procedures for uncertain attached equipment in large dynamical systems.

\section{Single longeron panel}

The first panel consists of a .04 inch thick aluminum skin with a single longitudinal stringer dividing the panel skin into two equal bays of 37.75 by 7.625 inches. A single line of thirty $6-32 \times$ $3 / 8$ screws attach the longeron to the panel skin. The panel is mounted in a rigid frame to simulate clamped boundary conditions. It is referred to as 
either the single longeron pancl, or the two-bay panel.

\section{Flat fuselage panel}

This second panel is representative of current aircraft construction but was manufactured without curvature to simplify the experimental and analytical modeling. It is constructed of a 47 - by 72 -inch aluminum panel with a 0.050 -inch skin with six equally spaced longerons and four equally spaced frame stiffeners. Single lines of rivets attach the stringers and frames to the skin. A bay is defined as a section of the panel skin that is bounded by the stringers and frames. The bay responses are the focus of the fuselage panel correlation efforts. For both the experimental and analytical studies on this panel, free-free boundary conditions were simulated.

\section{Progress in modeling}

Conventional FE modeling of the fuselage pane $1^{19,20}$ has focused on different stiffener to skin attachment models and their effects on the predicted bay motions. To characterize the panel dynamic response up to $1000 \mathrm{~Hz}$, several methods of modeling the panel were examined. First, the required finite element mesh density of the panel skin was evaluated by performing a normal mode analysis of a single bay with clamped boundary conditions. The skin was modeled with linear plate elements. $A$ mesh of 30 by 16 elements was found to provide a one-percent convergence on frequency and adequate spatial resolution to define the mode shapes through $1000 \mathrm{~Hz}$. This resulted in 11682 linear plate elements for the overall panel skin.

For the FSA study, the stiffeners were modeled using one-dimensional beam elements with the effective cross-sectional properties (area, inertia, and torsional constant) of the stiffeners. Offsets from the skin to the stiffener noutral axis are also included. The beam elements are created along a line consistent with the rivet line that attaches the skin to the stiffeners. The beam stiffener model of the panel contains approximately 60,000 degrees of freedom (DOFs). Similarly, the two-bay panel was modeled using a combination of linear plate elements for the skin and beam elements for the longeron. The resulting model of the two-bay panel has approximately 7900 DOFs.

The FSA modeling effort is currently underway. Preliminary results indicate that one must be very careful in picking appropriate values of fuzzy mass, fuzzy damping, and fuzzy modal density as input parameters for the FSA procedures. If these studies are successful, plans are underway to apply the FSA procedures to more complicated prototypical structures including NASA Langley's Aluminum Testbed Cylinder (ATC). ${ }^{21}$

\section{Conclusions}

This paper has described an implementation of Soize's fuzzy structures analysis (FSA) procedures in MSC/NASTRAN and MATLAB. The procedures have been validated by comparison to the results for a simply supported fuzzy beam already in the literature. Previous studies have shown that with FSA one can obtain similar results to a Monte Carlo simulation of uncertain attacliments with a greatly reduced computational expense. The work now underway is aimed at the fuzzy modeling of the skin attachments in two protolypical aircraft panels, and detailed results are expected soon.

\section{Acknowledgment}

Work supported by NASA Research Cooperative Agreement $\mathrm{NCC}-1-382$.

\section{Disclaimer}

Any opinions, findings, conclusions, or recommendations expressed in this publication are those of the authors and do not necessarily reflect the views of the National Aeronautics and Space Administration.

\section{$\underline{\text { References }}$}

1. See www.onera.fr,

2. C. Soize, "Probabilistic structural modeling in linear dynamic analysis of complex mechanical systems. I. Theoretical elements," La recherche Acrospatiale No. 1986-5, 23-48 (1986).

3. F. Chabas, A. Desanti, and C. Soize, "Probabilistic structural modeling in linear $\mathrm{dy}$ namic analysis of complex mechanical systems. II. Numerical analysis and applications," La recherche Aerospatiale No. 1986-5, 49-67 (1986).

4. C. Soize, "A model and numerical method in 
the medium frequency range for vibroacoustic predictions using the theory of structural fuzzy," J. Acoust. Soc. Am. 94, No. 2, Pt. 1, 849865 (1993).

5. C. Soize, "Vibration damping in low-frequency range due to structural complexity: A model based on the theory of fuzzy structures and model parameters estimation," Computers and Structures 58, No. 5, 901-915 (1995).

6. R. Ohayon and C. Soize, Structural Acoustics and Vibration (Academic Press, San Diego, 1998), particularly Chap. 15.

7. C. E. Ruckman and D. Feit, "A tutorial on Soize's method for stochastic modeling in structural acoustics (fuzzy structures analysis)," in DE-Vol. 84-2, Proceedings of the 1995 ASME Design Engineering Technical Conferences, Vol. 3B: Acoustics, Vibrations and Rotating Machines, pp. 241-246. Edited by R. Daddazio, M. Ettouney, N. Abboud, et al. ASME, New York (1995).

8. A. D. Pierce, D. Russell, and V. W. Sparrow, "Fundamental structural acoustic idealizations for structures with fuzzy internals," ASME J. Vib. Acoust., 117 339-348 (1995).

9. D. A. Russell and V. W. Sparrow, "Backscattering from a baffled finite plate strip with fuzzy attachments," J. Acoust. Soc. Am., 98 (3) 1527-1533 (1995).

10. A. D. Pierce, "Resonant frequency distribution of internal mass inferred from mechanical impedance matrices, with application to fuzzy structure theory," ASME J. Vib. Acoust., 119 324-333 (1997).

11. R. L. Weaver, "Mean and mean-square responses of a prototypical master/fuzzy structure," J. Acoust. Soc. Am. 101, No. 3, 1441-1449 (1997).

12. M. Strasberg and D. Feit, "Vibration damping of large structures induced by attached small resonant structures," J. Acoust. Soc. Am. 99, No. 1, 335-344 (1996).
13. MSC/NASTRAN, (MSC Software Corp., Los Angeles, CA.) See www.mscsoftware.com .

14. MATLAB, Ver. 5.0. (Mathworks Inc., Natick, MA). See www mathworks.com .

15. J. Cipolla, private communication, 1995; formerly with Naval Undersea Warfare Center, Division Newport; now with Hibbitt, Karlsson, \& Sorensen, Inc., Newport, R.I..

16. C. E. Ruckman "Preliminary report on implementing fuzzy structures analysis in NASTRAN," Carderock Division Naval Surface Warfare Center Rept. CDNSWC-SIG-94/142 (October 1994).

17. SARA (BBN Technologies, Cambridge, MA). See www.gte.com/ AboutGTE/ gto/ bbnt/ apts/ offerings/ sara.html .

18. V. W. Sparrow, D. A. Russell, J. D. Rochat, "Implementation of discrete fuzzy structure models in Mathematica," Int. J. Num. Meth. Engr. 37, 3005-3014 (1994).

19. G. A. Fleming, R. D. Buehrle, and O. L Storaasli, "Modal Analysis of an Aircraft Fuselage Panel Using Experimental and Finite Element Techniques," Procecdings of the 3rd International Conference on Vibration Measurements by Laser Techniques, Ancona, Italy, June 1998.

20. R. D. Buehrle, G. A. Fleming, R. S. Pappa, and F. W. Grosveld, "Finite Element Model Development and Validation for Aircraft Fuselage Structures," Proceedings of the 18th International Modal Analysis Conference, San Antonio, Texas, February 2000.

21. F. W. Grosveld, "Structural normal mode analysis of the aluminum testbed cylinder (ATC)," AIAA Paper 98-1949, 39th Structures, Structural Dynamics, and Materials Conf., April 20-23, 1998, Long Beach, CA. 


\section{$\underline{\text { Figures }}$}

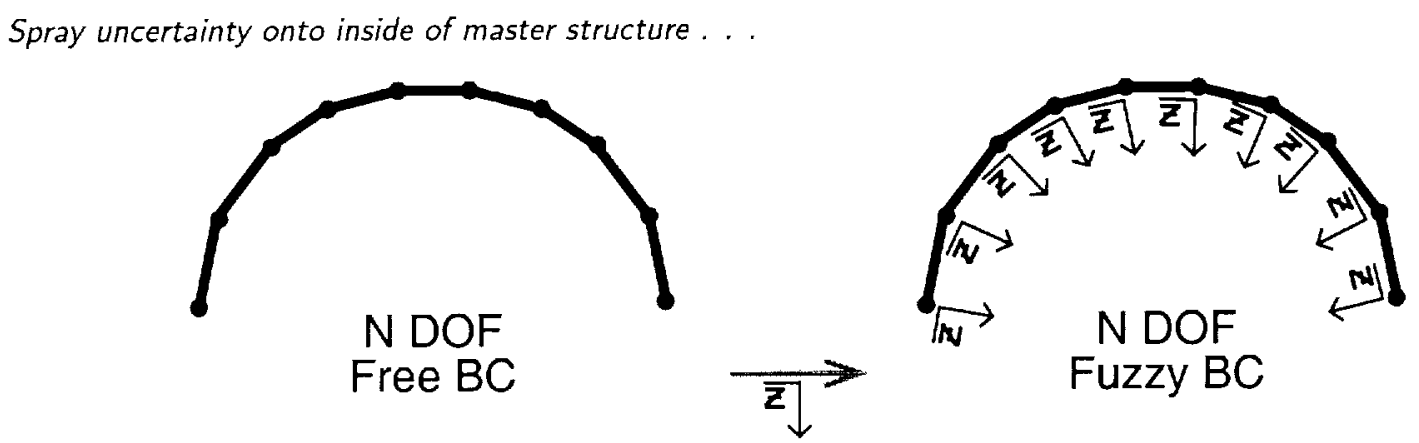

Figure 1: A FE model is made fuzzy by attaching appropriate boundary conditions to nodes. Each boundary condition is modeled as the impedance $Z$ of an attached 1-DOF oscillator. Note the the total number of DOFs in the model is unchanged when the fuzzy substructure is added.

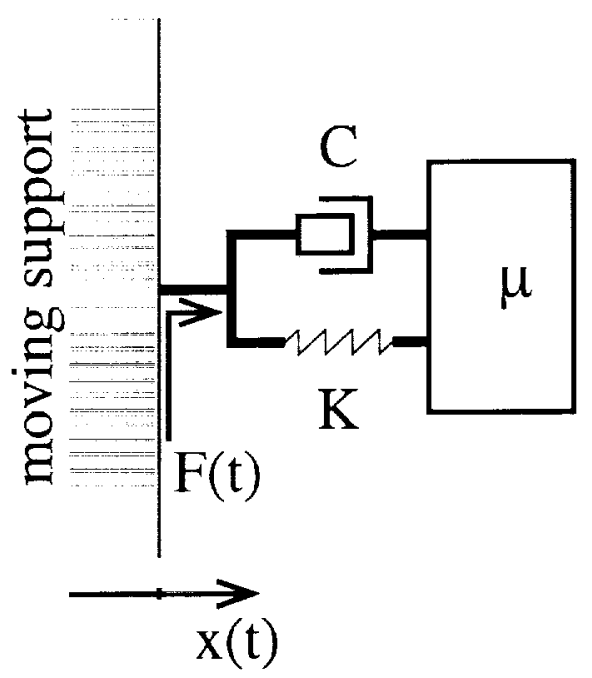

Figure 2: The 1-DOF model used for each fuzzy DOF of the fuzzy substructure. The moving support, or master structure, moves with displacement $x(t)$ and velocity $\dot{x}(t)=v(t)=\hat{v} e^{i \omega t}$. The resulting force due to the fuzzy 1-DOF attachment is $F(t)=\hat{F} e^{i \omega t}$. 


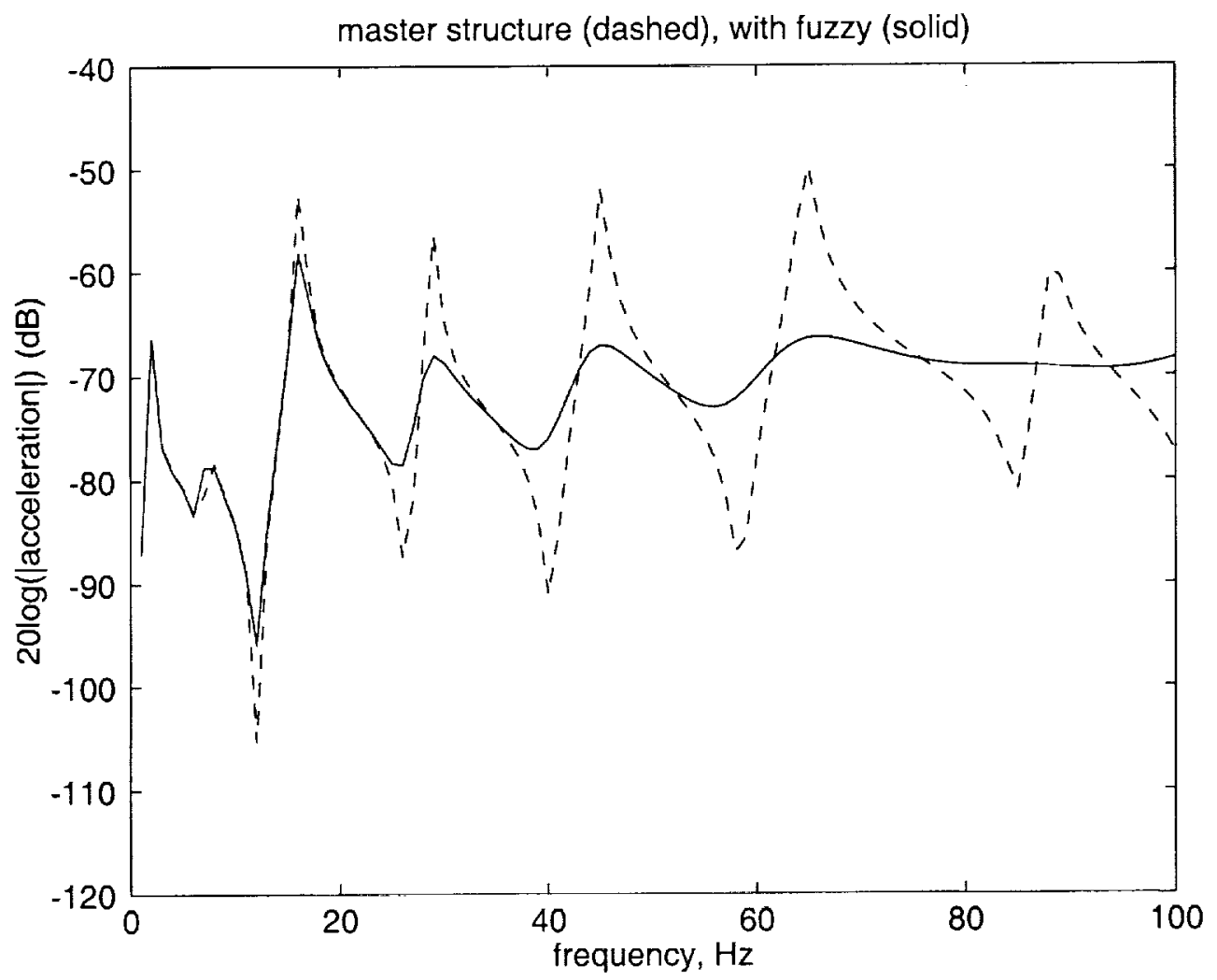

Figure 3: Mean squared acceleration at a point on Soize's simply supported beam versus frequency. The dashed line shows the result for the master structure alone, while the solid line shows the result including the effects of the fuzzy substructure. The horizontal axis is the frequency in hertz and the vertical axis is the expected mean squared acceleration in $\mathrm{dB}$ relative to $1 \mathrm{~m} / \mathrm{s}^{2}$. A $1 \mathrm{~Hz}$ frequency resolution is used. These results compare favorably to Fig. $7(\mathrm{a})$ of Soize's work. ${ }^{5}$ 